\title{
Unshackling caspase-7 for cancer therapy
}

\author{
Maria Eugenia Guicciardi and Gregory J. Gores
}

\author{
Division of Gastroenterology and Hepatology, College of Medicine, Mayo Clinic, Rochester, Minnesota, USA.
}

\begin{abstract}
Numerous solid tumors and hematologic malignancies acquire resistance to apoptosis-inducing chemotherapeutic drugs by downregulating the key effector caspase-3. These cells rely on caspase-7 to execute the apoptotic program, yet binding with XIAP constitutively inhibits active caspase-7 ( $\mathrm{p} 19$ / p12-CASP7). In this issue, Lin et al. describe how a newly synthesized drug is able to disrupt the XIAP:p19/p12-CASP7 complex and induce apoptosis in caspase-3-deficient cancer cells in vitro and in vivo. As this compound appears to exhibit minimal toxicity on normal tissues, it may represent a promising therapeutic agent to help treat caspase-3-deficient tumors.
\end{abstract}

\section{The challenge}

Apoptotic pathways activated either by engagement of cell surface death receptors (extrinsic pathways) or by intracellular stressors converging on mitochondria (intrinsic pathway) all result in the activation of effector caspases. The first and most active of the effector caspases is caspase- 3 , which activates caspase-7 and caspase- 6 via a cascade-like mechanism, ultimately resulting in the degradation of several intracellular substrates and cellular demise. Downregulation of caspase-3 is an effective apoptosis-evading mechanism frequently observed in cancer cells in association with acquired chemoresistance to apoptosis-inducing anticancer drugs. Indeed, re-expression of caspase-3 often restores sensitivity to apoptosis (1). In these cells, activation of the functionally similar caspase-7 may provide a viable therapeutic option to overcome apoptosis resistance. Previous studies have reported that upon engagement of the intrinsic apoptosis pathway (often triggered by chemotherapeutic drugs), caspase-7 is processed and activated and rapidly associates with X-linked inhibitor of apoptosis protein (XIAP) in a stable complex of approximately $200 \mathrm{kDa}$ (Figure 1A and ref. 2). This complex is formed regardless of the presence of caspase-3, but its inhibitory effect is particularly relevant in caspase-3-deficient cells, which rely on caspase- 7 as their major executioner caspase. Indeed, accumulation of the complex formed by binding of XIAP to active caspase-7 (p19/p12-CASP7) occurs in caspase-3-deficient cells after treatment

Conflict of interest: The authors have declared that no conflict of interest exists.

Citation for this article: J Clin Invest. 2013; 123(9):3706-3708. doi:10.1172/JCI71440. with chemotherapeutic drugs, and it is thought to be the main mechanism conferring chemoresistance to cancers with caspase-3 downregulation (2). Therefore, a therapeutic approach aimed to disrupt the XIAP:p19/p12-CASP7 complex may help restore sensitivity to apoptosis-inducing anticancer drugs in caspase-3-downregulated tumors.

\section{The strategy}

XIAP, like all inhibitor of apoptosis proteins (IAPs), contains three characteristic baculovirus IAP repeat (BIR) domains through which it interacts with different substrates. The inhibitory binding of XIAP to active caspase-3 and p19/p12-CASP7 occurs via a two-site interaction mechanism: first, the linker region between the BIR1 and BIR2 domains of XIAP interacts with the substrate-binding site of the activated caspases; and second, a surface groove on BIR2 then binds to the $\mathrm{N}$-terminal regions of activated caspase- 3 and caspase-7 (3). In this issue of the JCI, Lin et al. (4) identified $\mathrm{Cys}^{246}$ on caspase-7 as the critical residue for the protein-protein interaction (PPI) between p19/p12-CASP7 and XIAP, and generated a synthetic compound (iodoacetyl-lysine-BOC; I-Lys) capable of disrupting the XIAP:p19/p12-CASP7 complex by alkylating the Cys ${ }^{246}$ residue. Using the caspase-3-deficient breast cancer cell line MCF-7, the authors demonstrated that the XIAP:p19/p12-CASP7 complex is constitutively accumulated even in the absence of an apoptotic stimulus, and treatment with I-Lys induced dissociation of the complex with release of $\mathrm{p} 19 /$ p12-CASP7 and subsequent caspase-7dependent apoptosis (Figure 1B). Notably, accumulation of the XIAP:p19/p12CASP7 complex inversely correlated with caspase-3 expression in vitro and in vivo in samples from breast, colon, and lung cancers. XIAP expression was also found to negatively correlate with caspase-3 expression, but positively with $\mathrm{p} 19 / \mathrm{p} 12$-CASP7 accumulation. Conversely, accumulation of the XIAP:p19/p12-CASP7 complex was not detected in caspase-3-expressing cells, such as noncancerous MCF10A breast epithelium cells, which, in turn, did not undergo apoptosis in response to I-Lys. These observations suggest a specificity of I-Lys-induced apoptosis for caspase-3downregulated cancers, which was further confirmed in experiments utilizing reconstituted caspase- 3 expression in MCF-7 cells or caspase-3 knockdown in MCF10A cells. Activation of caspase-7 in nonapoptotic, caspase-3-deficient cells is likely due to its nonapoptotic functions, such as regulation of cell survival, proliferation, differentiation, and inflammation, for which caspases can be activated in the absence of the induction of an apoptotic cascade (5). Binding to XIAP therefore provides a safety mechanism to prevent the progression to apoptosis. In caspase-3-expressing cells, active caspase- 3 has been shown to cleave XIAP and promote its proteasomal degradation, reducing the binding of XIAP to p19/p12-CASP7 (Figure 1A and ref. 6). As I-Lys specifically targets the interaction between XIAP and p19/p12-CASP7, its use is limited to tumors with caspase- 3 downregulation and accumulation of the XIAP:p19/p12-CASP7 complex.

Since the role of IAPs in cancer development, progression, and chemoresistance has been established over the last two decades, there has been a growing interest in the development of small-molecule IAP inhibitors to be used in cancer therapy, either as single agents or in combination with other chemotherapeutic drugs (7). These compounds (named SMAC mimetics or IAP antagonists) mimic the IAP-binding motif of second mitochondria-derived activator of caspase/direct IAP-binding protein with low PI (SMAC/DIABLO), an endogenous IAP antagonist released from the mitochondria following activation of the intrinsic pathway of apoptosis. SMAC and its mimetics cause autoubiquitination and degradation 
A

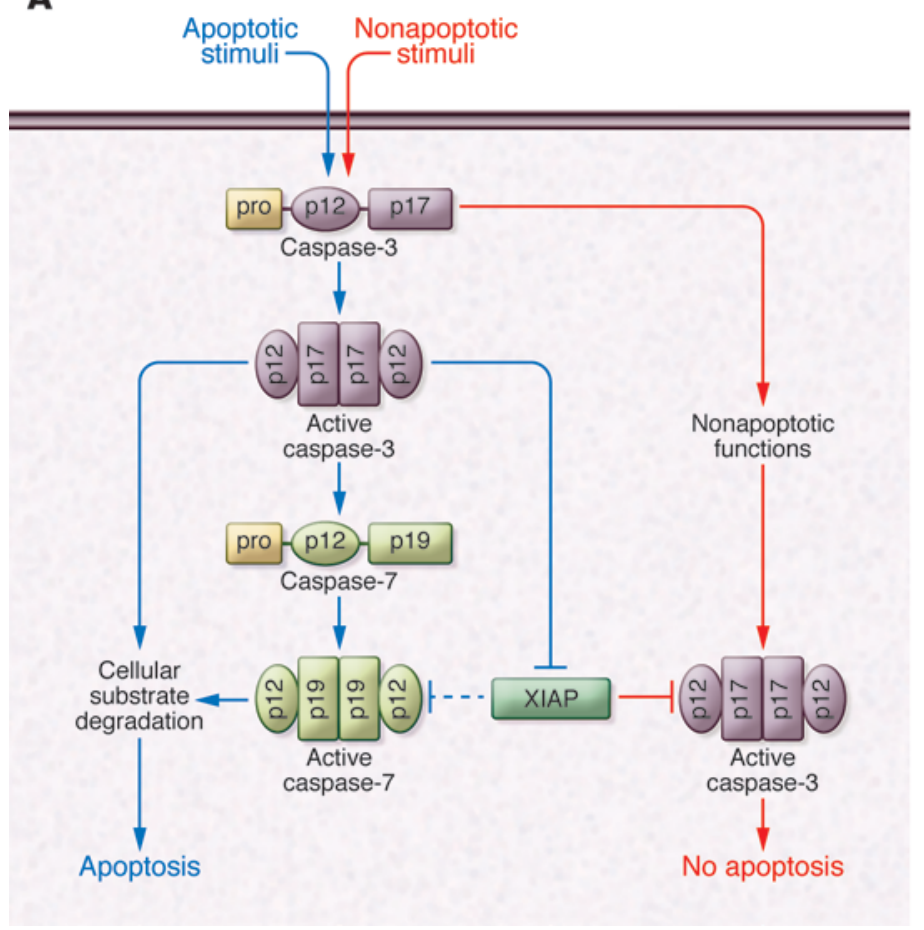

B

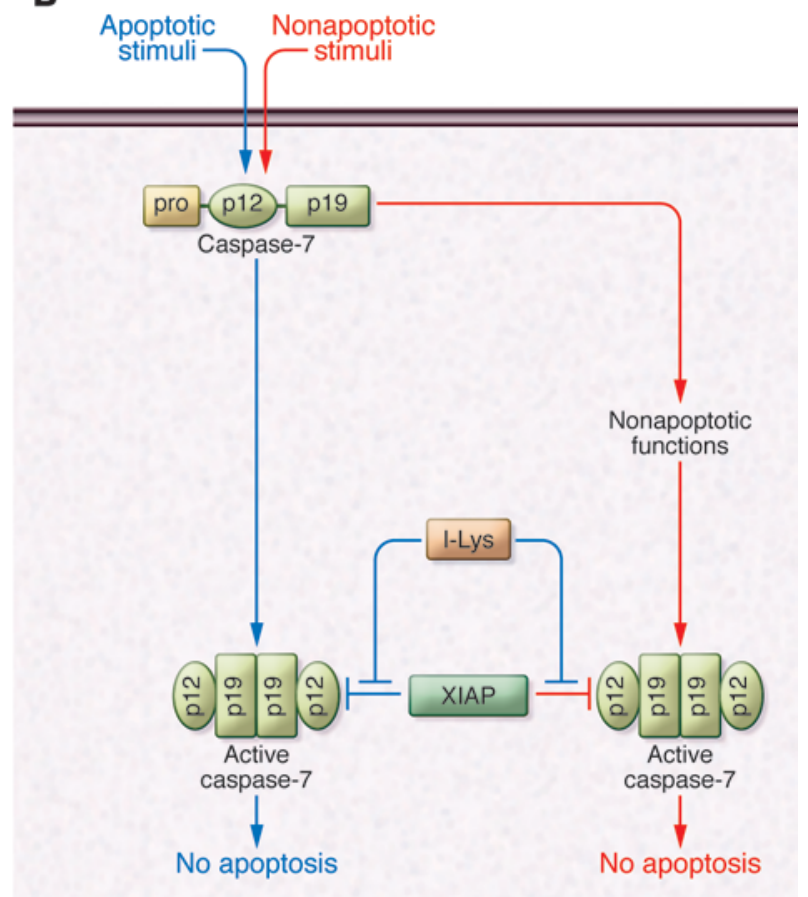

\section{Figure 1}

Both extrinsic and intrinsic apoptotic pathways result in activation of effector caspases. (A) Caspase-3 is the first of the effector caspases to be activated, which, in turn, proceeds to activate caspase-7 and caspase- 6 (not shown). Active caspase-3 also cleaves XIAP and promotes its degradation, preventing the inhibitory binding of XIAP to p19/p12-CASP7 or active caspase-3 itself. Active caspase-3 is also generated after nonapoptotic stimuli and is subsequently sequestered by XIAP to prevent unnecessary apoptosis. (B) In cells lacking caspase-3, apoptotic and nonapoptotic stimuli result in activation of caspase-7. Due to its high affinity to XIAP, p19/p12-CASP7 promptly binds to XIAP, restraining its apoptotic activity. The XIAP:p19/p12-CASP7 complex accumulates in caspase-3-deficient cells. Disrupting the XIAP:p19/p12-CASP7 complex using I-Lys or possibly other agents releases p19/p12-CASP7 from the complex and restores sensitivity to apoptosis.

of cIAP-1 and cIAP-2 and inhibition of the caspase-binding activity of XIAP. Lin et al. did not test SMAC mimetics in their study (4), although they could have potentially complemented their results with I-Lys. The authors note that the use of SMAC mimetics in vivo is discouraged due to reported toxic off-target side effects, such as inhibition of hematopoietic progenitor/stem cell growth, whereas I-Lys exhibited a high selectivity for caspase-3-deficient tumors, with no apparent toxicity on normal tissues, when used in tumor-bearing mice. However, several phase I/II clinical trials testing the applicability of chemically different SMAC mimetics as anticancer treatments have now indicated that these compounds are well tolerated, with only moderate transient lymphopenia and neutrophilia reported in a few patients (7). Therefore, SMAC mimetics may represent an alternative therapeutic option to I-Lys, if proven to be able to dissociate the XIAP:p19/ p12-CASP7 complex as well. It must be noted, though, that since the XIAP:p19/p12CASP7 complex seems to form even in the presence of endogenous SMAC, it is possible that small-molecule SMAC mimetics would fail to disrupt the complex (2).

The molecular mechanisms behind downregulation of caspase- 3 in cancers are not well studied. Except in rare cases, such as MCF-7 cells, in which a 47-base pair deletion in CASP3 has been identified (8), the reason for loss of caspase- 3 is generally unknown. Recently, several studies have proposed a direct posttranscriptional regulation of caspase- 3 expression by microRNAs (miRNAs) (9-11). In particular, overexpression of miR155 has been associated with downregulation of caspase- 3 and resistance to apoptosis in the caspase-3-deficient breast cancer cell line MDA-MB-157 (10, 11). In their study, Lin et al. demonstrated that upregulation of another miRNA, let-7a-1, decreases caspase- 3 expression and is associated with significant accumulation of the XIAP:p19/ p12-CASP7 complex and enhanced chemoresistance (4). These findings suggest that, in a subset of tumors where caspase-3 downregulation is controlled by miRNAs, silencing the responsible miRNAs could potentially prevent the formation of the XIAP:p19/p12-CASP7 complex.

Overall, the study by Lin et al. provides important insights on the mechanisms underlying apoptosis resistance in caspase-3deficient tumors and highlights the need to interfere with the formation of the XIAP:p19/ p12-CASP7 complex in order to restore sensitivity to apoptosis-inducing drugs.

\section{Therapeutic implications}

The main concern in the design of a new anticancer therapy is its ability to selectively kill cancer cells without damaging normal tissues. Lin and coworkers went on to demonstrate the efficacy of I-Lys in the treatment of tumors in vivo in immunodeficient mice subcutaneously inoculated with caspase-3-null or caspase-3-expressing breast cancer cells (4). As expected, I-Lys treatment successfully inhibited caspase-3null tumor growth, but failed to do so in caspase-3-expressing tumors. Importantly, no pathological alterations were reported 
in the liver, heart, kidney, lung, and spleen of treated mice, which suggests that treatment with I-Lys could represent a specific and safe therapeutic strategy against caspase-3-downregulated tumors. The authors also demonstrated that low doses of I-Lys, incapable of inducing apoptosis as a single agent, synergistically increased sensitivity to chemotherapy-induced cell death in multidrug-resistant cancer cells. Despite these promising findings, more rigorous preclinical studies are still needed to establish whether I-Lys could be used in human cancer therapy. To turn I-Lys into a pharmacological agent for clinical use will require more in-depth analysis of its pharmacokinetics, bioavailability, and tolerability. Nonetheless, the present study by Lin et al. (4) provides a strategy for precision medicine, namely the biomarker of caspase- 3 deficiency, and suggests a novel approach to treat such tumors.

\section{Acknowledgments}

The secretarial assistance of Courtney Hoover is greatly appreciated.

Address correspondence to: Gregory J. Gores, College of Medicine, Mayo Clinic, 200 First Street SW, Rochester, Minnesota 55905, USA. Phone: 507.284.0686; Fax: 507.284.0762;

E-mail: gores.gregory@mayo.edu.

1. Devarajan E, et al. Down-regulation of caspase 3 in breast cancer: a possible mechanism for chemoresistance. Oncogene. 2002;21(57):8843-8851.

2. Twiddy D, Cohen GM, Macfarlane M, Cain K. Caspase-7 is directly activated by the approximately $700-\mathrm{kDa}$ apoptosome complex and is released as a stable XIAP-caspase-7 approximately $200-\mathrm{kDa}$ complex. J Biol Chem. 2006;281(7):3876-3888.

3. Scott FL, Denault JB, Riedl SJ, Shin H, Renatus M, Salvesen GS. XIAP inhibits caspase- 3 and -7 using two binding sites: evolutionarily conserved mechanism of IAPs. EMBO J. 2005;24(3):645-655.

4. Lin Y-F, et al. Targeting the XIAP/caspase-7 complex selectively kills caspase-3-deficient malignancies. J Clin Invest. 2013;123(9):3861-3875.

5. Lamkanfi M, Festjens N, Declercq W, Vanden Ber- ghe T, Vandenabeele P. Caspases in cell survival, proliferation and differentiation. Cell Death Differ. 2007;14(1):44-55

6. Hornle M, Peters N, Thayaparasingham B, Vorsmann H, Kashkar H, Kulms D. Caspase-3 cleaves $\mathrm{XIAP}$ in a positive feedback loop to sensitize melanoma cells to TRAIL-induced apoptosis. Oncogene. 2011;30(5):575-587.

7. Fulda S, Vucic D. Targeting IAP proteins for therapeutic intervention in cancer. Nat Rev Drug Discov. 2012;11(2):109-124.

8. Janicke RU, Sprengart ML, Wati MR, Porter AG. Caspase- 3 is required for DNA fragmentation and morphological changes associated with apoptosis. J Biol Chem. 1998;273(16):9357-9360.

9. Quintavalle C, et al. Effect of miR-21 and miR$30 \mathrm{~b} / \mathrm{c}$ on TRAIL-induced apoptosis in glioma cells [published online ahead of print September 10, 2012]. Oncogene. doi: 10.1038/onc.2012.410.

10. Zhang C, Zhao J, Deng H. 17beta-Estradiol up-regulates miR-155 expression and reduces TP53INP1 expression in MCF-7 breast cancer cells. Mol Cell Biochem. 2013;379(1-2):201-211.

11. Zheng SR, Guo GL, Zhai Q, Zou ZY, Zhang W. Effects of miR-155 antisense oligonucleotide on breast carcinoma cell line MDA-MB-157 and implanted tumors. Asian Pac J Cancer P. 2013; 14(4):2361-2366. 